\title{
Emotion based Music Retrieval for Gesture Recognition
}

\author{
G.Deepa \\ M.Tech,Student, \\ DECS, Dept. of ECE \\ Vardhaman College of Engineering, \\ Hyderabad, INDIA
}

\author{
G. Bhaskar Phani Ram \\ Assistant Professor, \\ Dept. of ECE \\ Vardhaman College of Engineering, \\ Hyderabad, INDIA
}

\begin{abstract}
Emotion based music retrieval is a music player based on user's emotion. Many music devices and mobile music players are used to listen to music. A practical problem is selection of desired music. Nowadays many devices are integrated with cameras. This paper gives how to take advantage of these one camera systems. In this proposed system emotion is derived automatically from hand gestures where hand gestures are captured by camera. The data recorded from hand gestures are coupled with musical emotion. Users can search through music collection based on emotional character of music which expressed from hand gestures. The main aim of this project is to design emotion based user interface for music retrieval. The algorithms were implemented in the system using $\mathrm{C}++$. The system uses the Intel OpenCV library for image processing. This paper is implemented by using ARM9 micro controller. The camera is connected to the controller through USB device. The microcontroller is connected to PC through serial communication. The controller processes the information and monitors the results as controlled songs on PC.
\end{abstract}

\section{Keywords}

Gesture Recognition, Human Computer Interface, Music Retrieval, Image Processing, Emotion Recognition, OpenCV.

\section{INTRODUCTION}

Music plays very important role in our daily life. Music and emotion are strongly related. Many music players are used to listen to music in our daily activities. A practical problem is selection of desired music from music collection. The main aim of this proposed system is emotion based user interface for music retrieval. Here emotion is expressed through hand gestures. These hand gestures are captured by camera. The data recorded from hand gestures are coupled with musical emotion. So users can search music based on emotional character of music which is expressed from hand gestures. Gesture is a movement of part of the body to express an idea or feeling. Emotions are expressed by hand gesture. There are two types of gestures (i) Static gesture (ii) Dynamic gesture. Static hand gesture is a posture of hand. Dynamic gesture is a movement of hand. Gesture recognition is a technology that achieves human-machine interactions that do not require contact based input mechanisms like remote, mouse, keyboard etc.

Work on hand gesture recognition has been reported several fields of research. Vision based hand gesture recognition [3] uses only webcam and computer vision technology such as image processing that can recognize several hang gestures. Computer vision methods for hand gesture interfaces [4] must better than current performance in terms of robustness and speed to achieve interactivity and usability. Interface to interact with physical world [5] implemented using hand gesture recognition and object detection using OpenCV and information related to detect objects. Gesture Controlled MP3 player [7] developed using image processing in mat lab and edge detection algorithm. E-Motion based music retrieval [1] presented a foundation for a motion based user interface for music retrieval using wireless handheld device equipped with inertial sensors [9]. A system is developed for controlling music player [2] using the webcam for capturing the hand gestures, identifying them and finally accessing the music player. Fast and efficient method for finger tip detection [10] [11] developed and the movement of user's finger will control the robot hand and its working, by moving hand in front of camera without wearing any gloves or markers.

\section{SYSTEM OVERVIEW}

This system deals with a method to recognize hand-gesture. The system uses single camera to recognize the user's handgesture. It is very hard to recognize hand gesture since a human hand is the object with high degree of freedom, the well-known problem in vision-based recognition area However, when we use OpenCV library to detect a frontal hand in an image using its Haar Cascade, this will increase the human computer interaction by using real time hand gestures. The data recorder from hand gestures are coupled with musical emotion. Phonon multimedia framework is used to assign music to hand gestures. So that when user place hand in front of camera the particular song assigned to hand gesture is played through PC.

This system supports feature of image/video processing by using various algorithms and features using image processing. In our designed system before playing the songs, we have to load list of songs. After that we have to place our hand in front of the camera. The camera will capture the hand movement and given it to controller. The controller will estimate the image and extract the foreground from the image and track music of image. The controller will send this information PC through serial communication. Based on the hand movement we can play the songs. Here we are using ARM micro controller. This project is based on Linux operating system (Ubuntu Linux).

OpenCV is an open source computer vision library which runs under Linux, Windows, Mac OS X and it is written in C and $\mathrm{C}++$. OpenCV was designed for computational efficiency and with a strong focus on real-time applications. OpenCV library provides us a greatly interesting demonstration for hand gesture detection. Furthermore it provides us functions that they used to train classifiers for their hand detection system, called Haar-Training, so that we can create our own object classifiers using these functions. 


\section{WORKING MECHANISM}

In this concept the music players are controlled using by gestures. An input is taken from camera and image will be processing in ARM controller using image processing techniques and recognize hand gestures. After recognizing hand gestures the result given to the PC. RS232 it communicates the signals from ARM9 controller to PC. The block diagram shown in Figure 1.Power supply is needed from $+5 \mathrm{v}$ to $+12 \mathrm{v}$ to the ARM controller using transformer, bridge rectifier, filters and regulators.
ARM9 is an ARM architecture 32-bit RISC family. With this design generation, ARM moved from von Neumann architecture (Princeton architecture) to Harvard architecture with separate instruction and data busses, significantly increasing its potential speed. Most silicon chips integrating these cores will package them as modified Harvard architecture chips, combining the two address busses on the other side of separated CPU caches and tightly coupled memories.

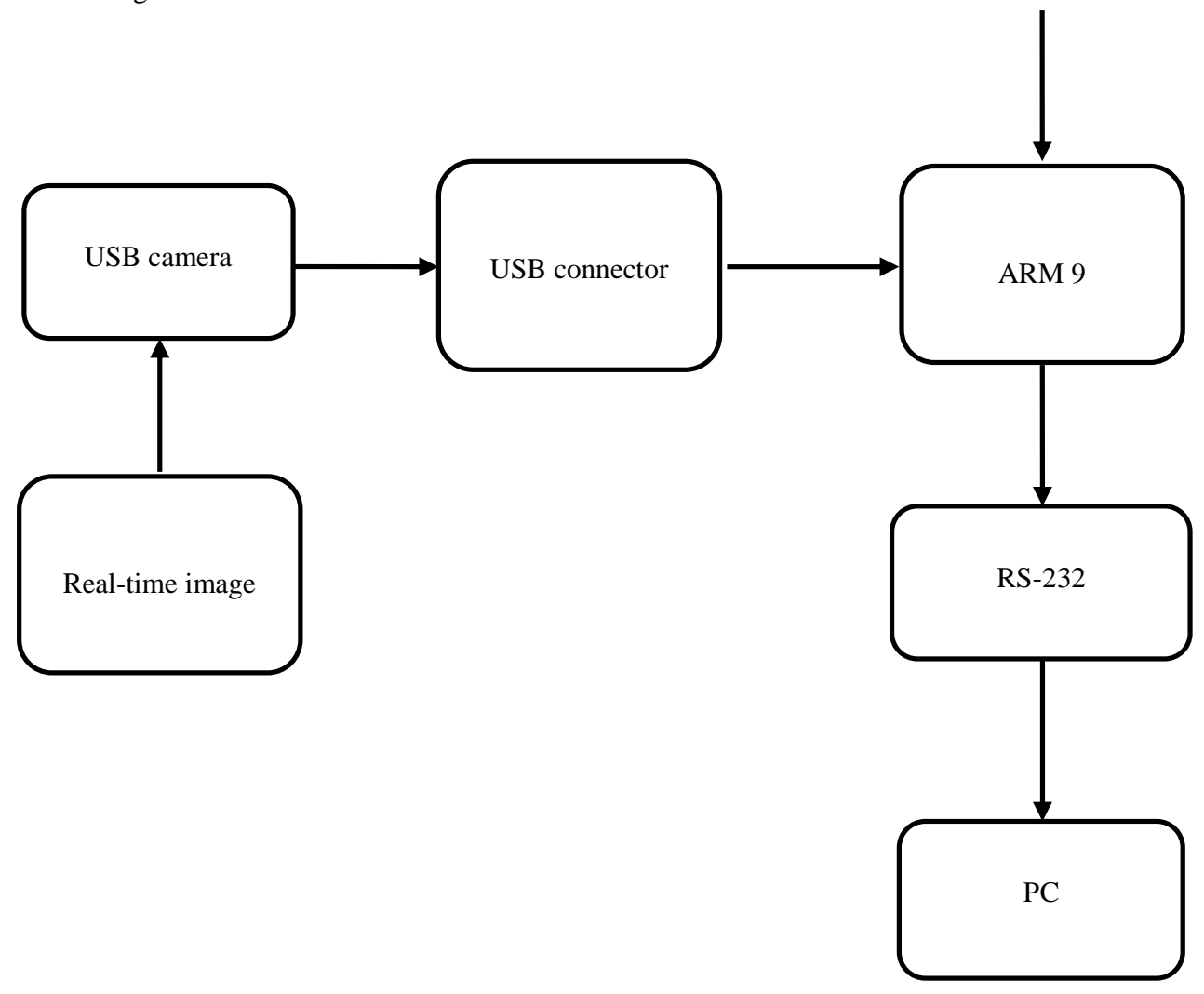

Figure 1: Block diagram

\section{PROPOSED ALGORITHM}

This paper proposes an algorithm for the real-time static recognition of hand gestures for HCI. This real-time algorithm is based on two phases of implementation- the hand gesture recognition phase and track music of hand gesture phase.This system uses linux operating system, opencv library and Haar classifier. The proposed system shown in Figure 2.

In this particular implementation the live input real-time image captured through camera. Thus any potential gesture or movement made in front of camera from this point onwards will be processed for recognition.

The first phase of processing, hand gesture recognition, is carried out by using the Haar cascade hand recognizer. In this proposed system hand gesture recognition is depends on position of hand gesture in the image like left, right, top and bottom. We assigned musical emotion for particular position of hand gesture like angry, relax, happy and sad. Phonon multimedia framework is used to assign music for hand gestures. The second phase of processing, track music of hand gesture, after recognizing position of real-time hand gesture the

particular music which assigned to hand gesture is played through PC.
The Haarcascades training(haar training) is a quick tool to achieve accurate hand gesture detection and recognition. The face, hand and body detection examples included in OpenCV installation example folder(/opencv/data/haarcascades/) demonstrate how fast haarcascade files help to do the job. In this proposed algorithm haarcascades training file haarcascade_hand.xml is used for hand gesture detection.

Phonon is a cross-platform multimedia frame network that enables the use of audio and video content in Qt applications. Qt is a cross-platform application framework that is widely used for developing application software with a graphical user interface (GUI), and also used for developing non-GUI programs such as command-line tools and consoles for servers. Qt uses the phonon multimedia framework to provide functionality for playback of the most common multimedia formats. The playback is started and managed by a media object, which sends the media stream to any sinks connected to it by a path. The sink (I/O device) then plays the stream back, usually through a sound card. 


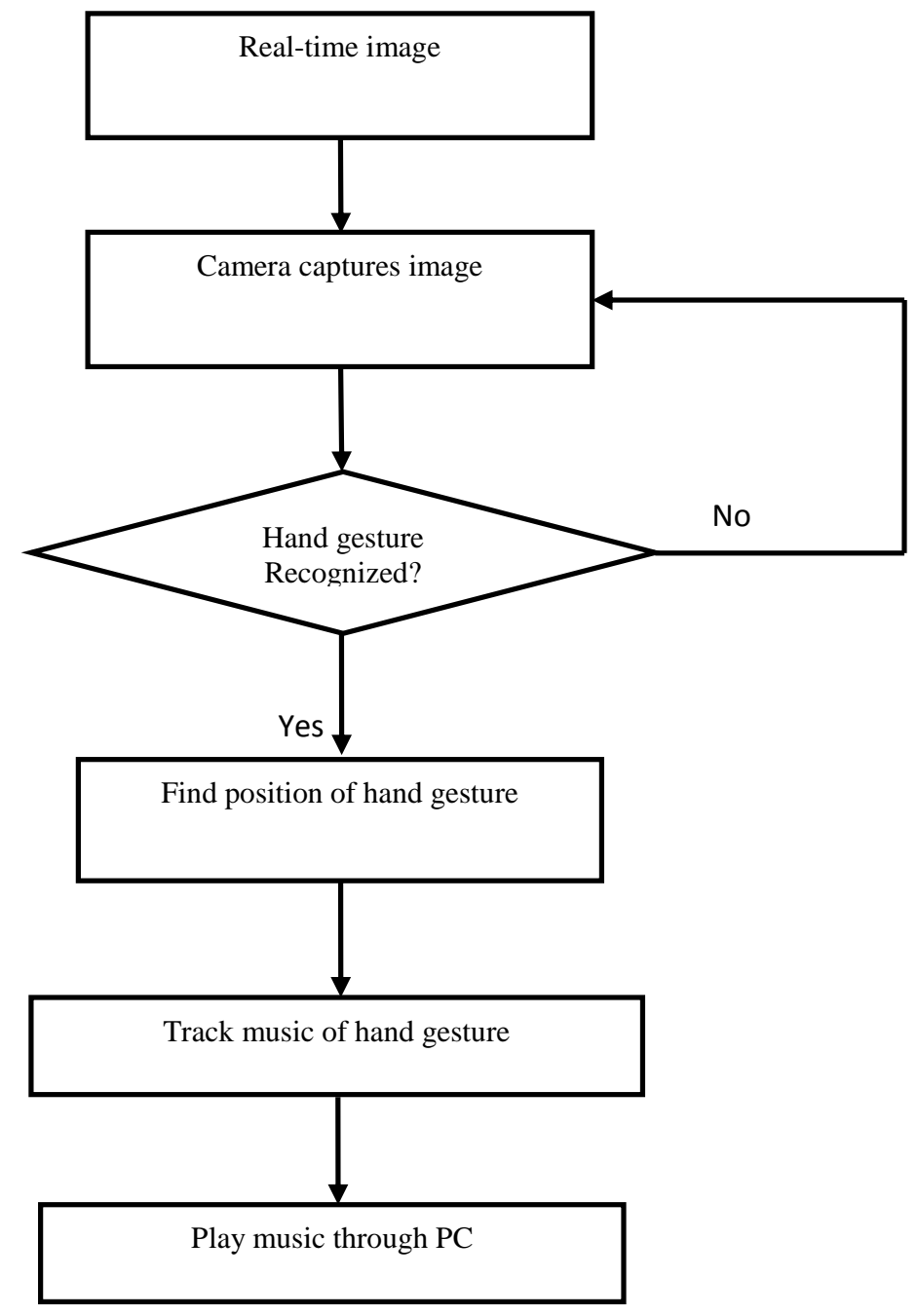

Figure 2: Proposed system

\section{EXPERIMENTS AND RESULTS}

Camera is used to capture the real-time image. Here we draw a rectangle inside the image using OpenCV library. The camera display shown in figure 3 . Here we assign happy music for the hand gesture above the rectangle. So if the users hand gesture is above the rectangle happy music will play automatically as sown in figure 4. Similarly for the hand gesture below the rectangle here assigns sad music. If the user shows hand gesture below the rectangle it will play sad music as shown in figure 5 .
The angry music is assigned for the hand gesture left side of the rectangle. So users can play angry music by showing the hand gesture left side of the rectangle as shown in figure 6.The relaxation music is assigned to the hand gesture which shows the right side of the rectangle. So users can play relaxation music by showing the hand gesture right side of the rectangle as shown in figure 7 . 


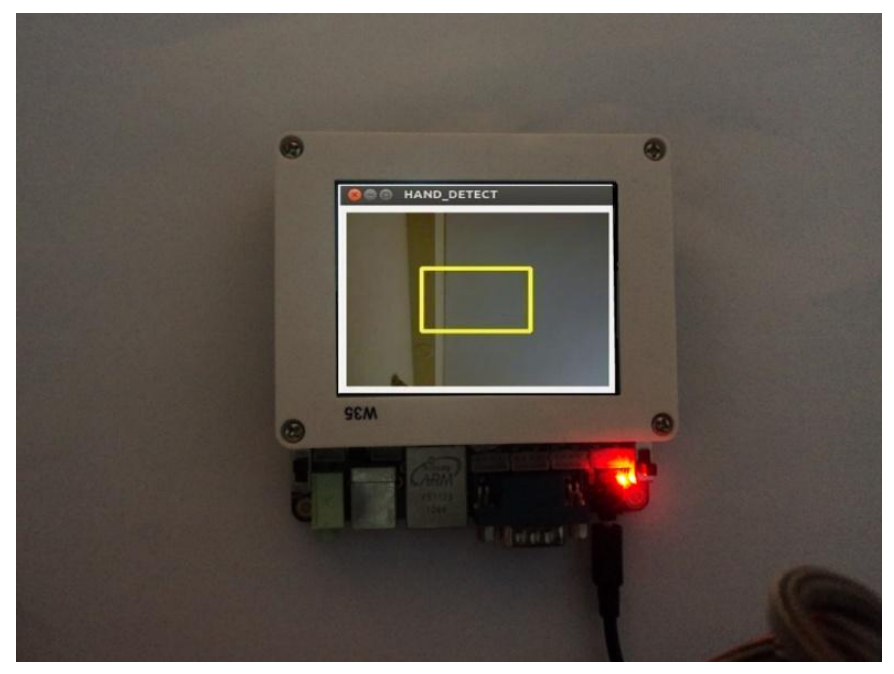

Figure 3: Camera display

\section{QQD HAND_DETECT}

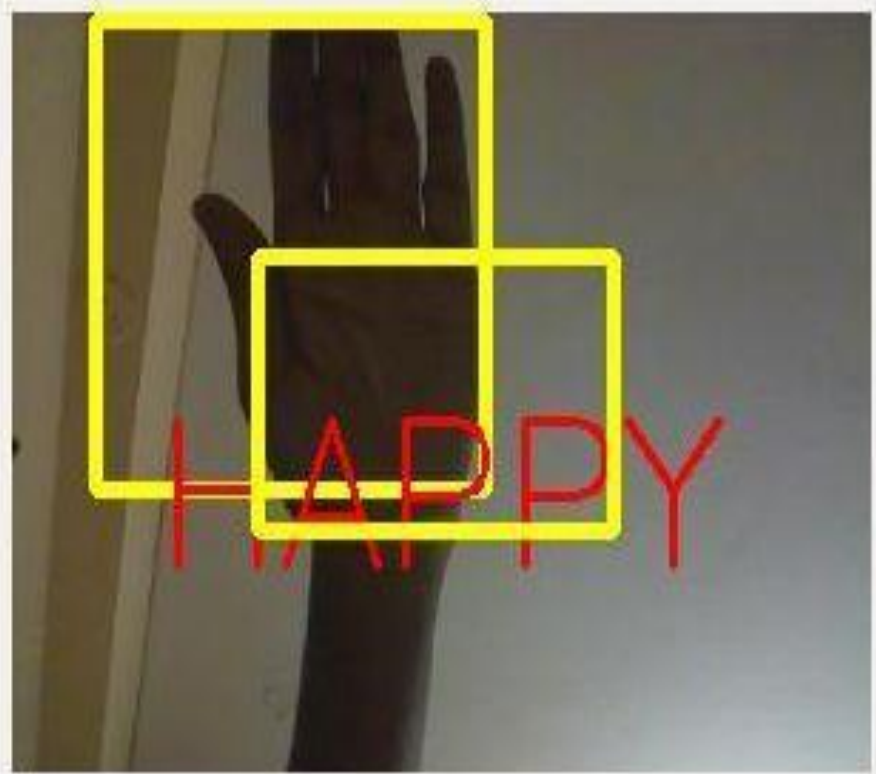

Figure 4: Happy 


\section{QQQ HAND_DETECT}

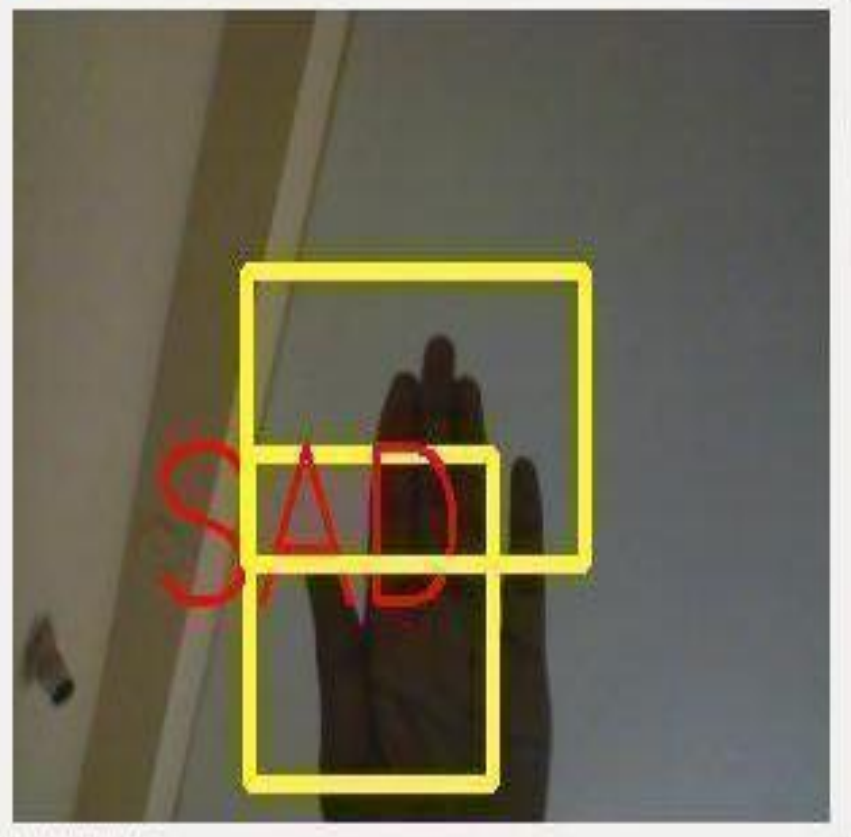

Figure 5: Sad

\section{Q 0 Q HAND_DETECT}

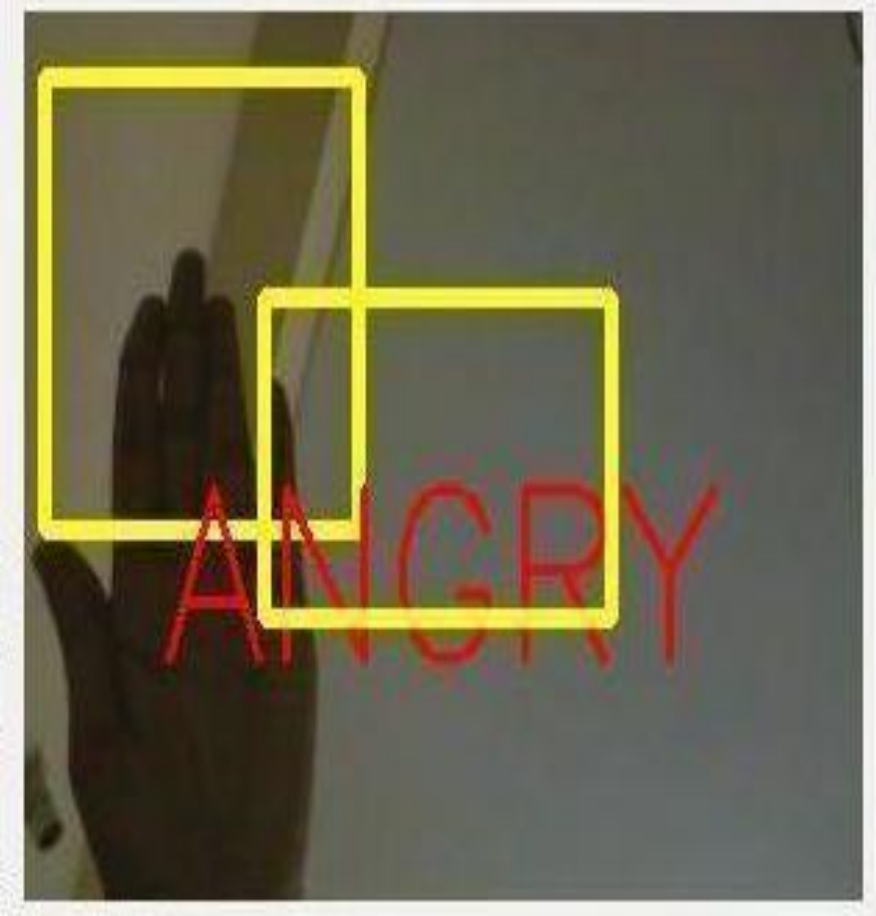

Figure 6: Angry 


\section{QQQ HAND_DETECT}

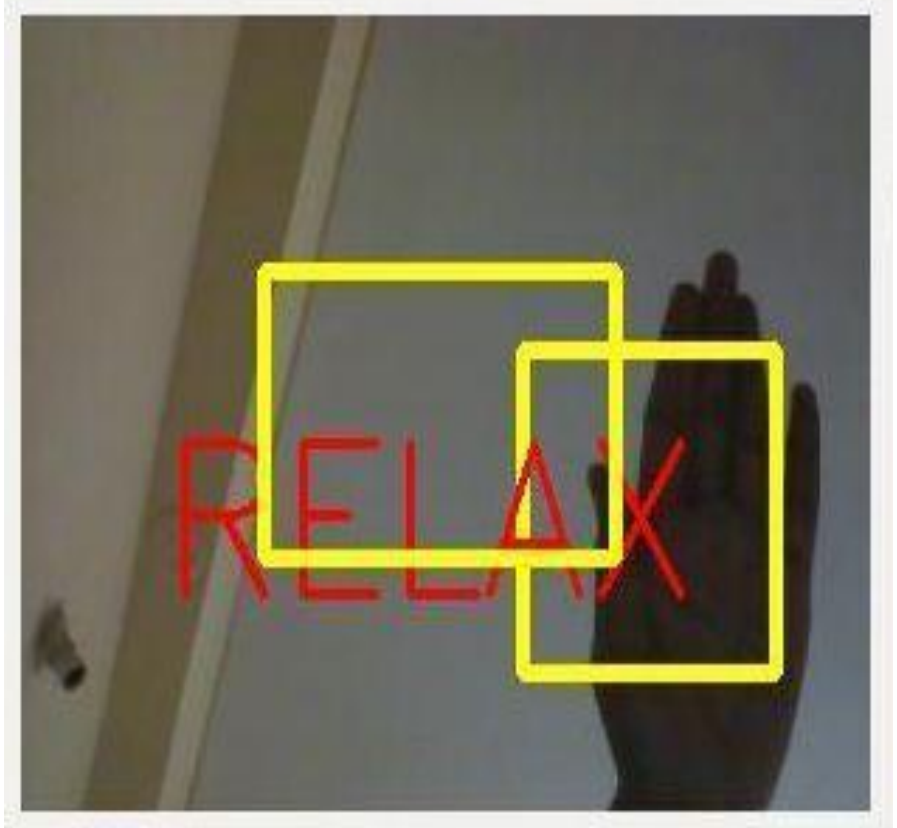

Figure 7: Relax

\section{CONCLUSION}

In the present world many ways are available for providing input to the system. Many of them require physical touch and some requires physical objects like remote, mouse, and keyboard. But very few applications are available that controlled and working with using hand gesture. By using hand gesture recognition user can interact with system without any physical touch. In this paper we have developed a system to control music based on user's emotion where emotion can be expressed through hand gestures.

\section{REFERENCES}

[1] Denis Amelynck, Marc Leman, Leon Van Noorden, Maarten Grachten and, "Toward E-Motion Based Music Retrieval a Study of Affective Gesture Recognition" IEEE Transactions on Affective Computing, vol.3, no.2, April-June 2012.

[2] Abim Pal, Hitesh Kumar and K.John Singh, "Music Controller Based On Hand Gestures Using Webcam", International Journal on Computer Science and Engineering, vol.4, no.11, Nov 2012.

[3] Radhika Bhatt, Nikita Fernandes, Archana Dhage, "Vision Based Hand Gesture Recognition for Human Computer Interaction", International Journal of Engineering Science and Innovative Technology, volume 2, issue 3, May 2013.

[4] Pallavi Halarnkar, Sahil Shah, Harish Shah, Hardik Shah, Jay Shah, "Gesture Recognition Technology: A Review" International Journal of Engineering Science and Technology, vol.4, no.11, November 2012.
[5] Prof. Suhas M.Patil, Nandkishor P.More, Sheetal S. Thokal, Aniket S.More, Bhagyavati V.Patil "Interface to Interact with Physical World using Human Gesture"

[6] International Jornal of Innovative Research in Computer and Communication Engineering, vol.1, Issue 2, April 2013.

[7] Tao Li and Mistunori Ogihara, "Toward Intelligent Music Information Retrieval" IEEE Transactions on Multimedia, vol.8, no.3, June 2006.

[8] Kurma Soujanya, Mr.M.Firdouse Ali Khan, "Design and Development of Gesture Controlled MP3 Player Using ARM7 and Image Processing Technique", International Journal of Research in Computer and Communication Technology, vol.2, Issue 1, January 2013.

[9] Lie Lu, Dan Liu, and Hong-jiang zhang, "Automatic Mood Detection and Tracking of Music Audio Signals", IEEE Transactions on Audio, Speech, and Language Processing, vol.14, no.1, January 2006.

[10] Nguyen Thuy Le, "EmuPlayer: Music Recommendation System Based on User Emotion Using Vital Sensor".

[11] M.K. Bhuyan Debanga Raj Neog and Mithum Kumar Kar, "Fingertip Detection for Hand Pose Recognition", International Journal on Computer Science and Engineering, vol.4, no.3, March 2012.

[12] Jagdish Lal Raheja, Karen Das, Ankit Chaudhary, "Fingertip Detection: A Fast Method with Natural Hand", International Journal of Embedded Systems and Computer Engineering, vol.3, no.2, July-December 2011. 